ESAIM: PROCEEDINGS AND SURVEYS, September 2014, Vol. 45, p. 437-446

J.-S. Dhersin, Editor

\title{
SOME CHARACTERIZATIONS OF A UNIFORM BALL PROPERTY*
}

\author{
JÉRÉMY DALPHIN ${ }^{1}$
}

\begin{abstract}
In 1973, Helfrich suggested a simple model to describe the shapes of vesicles: a free bending energy involving geometric quantities like curvature. However, the mathematical questions concerning the existence and the regularity of minimizers to such shape optimization problems still remain open. In this article, we consider a class of admissible shapes in which the existence of minimizers is ensured: the hypersurfaces of $\mathbb{R}^{n}$ satisfying a uniform ball condition. We prove that this property is equivalent to the notion of positive reach introduced by Federer in 1959. Then, another characterization in terms of $C^{1,1}$-regularity is established for compact hypersurfaces.

Résumé. En 1973, Helfrich proposa un modèle simple pour décrire les formes prises par les vésicules : une énergie élastique faisant intervenir des quantités géométriques comme la courbure. Cependant, les questions mathématiques liées à l'existence et la régularité de solutions pour de tels problèmes d'optimisation de formes restent ouvertes à ce jour. Dans cet article, on considère une classe de formes admissibles au sein de laquelle l'existence d'un minimum est assurée : les hypersurfaces de $\mathbb{R}^{n}$ qui vérifient une condition de boule uniforme. On démontre que cette propriété est équivalente à la notion de "reach" positif introduite par Federer en 1959. Ensuite, une autre caractérisation en termes de régularité $C^{1,1}$ est établie pour les hypersurfaces compactes.
\end{abstract}

\section{INTRODUCTION}

In biology, when a sufficiently large amount of phospholipids is inserted in a aqueous media, they immediately gather in pairs to form bilayers also called vesicles. Hence, a vesicle simply represents a bag containing a fluid and itself contained in a fluid. It is the basic membrane of all living cells and understanding it well is a first fundamental step in the comprehension of general cells behaviour.

Devoid of nucleus among mammals, red blood cells are typical examples of vesicles on which is fixed a network of proteins playing the role of a skeleton inside the membrane. In his thesis, the author is mainly interested in the shape of such vesicles using the optimization point of view.

It follows from the least action principle that the shape is minimizing a certain energy under some constraints. During the 70s, Canham in [1] and then Helfrich in [2] suggested a simple model to characterize vesicles. Imposing the area of the bilayer and the volume of fluid it contains, their shape is a minimizer for the following free bending energy:

$$
\mathcal{E}=k_{b} \int_{\text {membrane }}\left(H-H_{0}\right)^{2} d S+k_{g} \int_{\text {membrane }} K d S,
$$

\footnotetext{
* For his first article, the author wishes to express his thanks to his two PhD advisors: Antoine Henrot and Takeo Takahashi.

${ }^{1}$ Institut Elie Cartan of Lorraine UMR 7502, University of Lorraine BP 7023954506 Vandoeuvre-les-Nancy Cedex, France. e-mail: jeremy.dalphin@mines-nancy.org
}

(C) EDP Sciences, SMAI 2014 
where $d S$ is the infinitesimal area element corresponding to an integration with respect to ordinary 2-dimensional Hausdorff measure, $H=\frac{\kappa_{1}+\kappa_{2}}{2}$ the scalar mean curvature, $K=\kappa_{1} \kappa_{2}$ the Gaussian curvature, $H_{0}$ a fixed real number measuring the asymmetry between the two layers, and where $k_{b}$ and $k_{g}$ are two physical constants.

The mathematical questions at stake here are the existence of solutions to such shape optimization problems and the determination of an accurate class of admissible shapes. In calculus of variations, a minimizing sequence is considered and one has to introduce the right topology in order to fulfil both of these two contradictory objectives: compactness of the sequence and lower semi-continuity of the functional.

Geometric measure theory allows one to get a minimizer for the Helfrich energy: a possibly irregular object called current. However, numerical simulations from physicists like Seifert (see e.g. [3]) suggest a better regularity of the minimizer and it remains an open problem to understand precisely in which sense it is.

The 2D case has been considered by Bellettini and al. in [4], then recently used by Choksi and Veneroni in [5] to solve the axisymmetric situation in 3D. The Willmore functional $\left(H_{0}=0\right)$ has also been widely studied by geometers using its conformal invariance property: Willmore himself in [6], Simon in [7] for genus one, Bauer and Kuwert in [8] for higher genus, then recently Schygulla in [9] for analytic surfaces of genus zero.

In the general case $H_{0} \neq 0$, the regularity of minimizers remains an open problem and experiments show that singular behaviours can occur to vesicles such as budding effects. However, it cannot happen to red blood cells because their skeleton prevents the membrane from bending too much locally.

To take this aspect into account, we introduce a more reasonable class of admissible shapes formed by surfaces that satisfy a uniform ball condition. Generalizing what Chenais did in [10] when she considered the uniform cone property, we prove in a forthcoming paper [11] that in this class of objects, the existence of a minimizer is ensured for general functionals and constraints involving the second-order geometric properties of surfaces.

In this article, the $\varepsilon$-ball condition is first defined and showed to be equivalent to positiveness of the reach, a notion introduced by Federer in [12]. Then, another characterization in terms of $C^{1,1}$-regularity of surfaces is established in the framework of shape optimization, where a similar result has been obtained recently in [13]. The main results are precisely stated on page 4 in Theorem 1.6, Corollary 1.7, Theorem 1.8, and Theorem 1.9.

\section{Definitions, notation, and main Results}

Consider a natural number $n \geqslant 2$ henceforth set. The space $\mathbb{R}^{n}$ whose points are marked $\mathbf{x}=\left(x_{1}, \ldots, x_{n}\right)$ is naturally provided with its usual euclidean structure:

$$
\forall(\mathbf{x}, \mathbf{y}) \in \mathbb{R}^{n} \times \mathbb{R}^{n}, \quad\langle\mathbf{x} \mid \mathbf{y}\rangle=\sum_{k=1}^{n} x_{k} y_{k} \quad \text { and } \quad\|\mathbf{x}\|=\sqrt{\langle\mathbf{x} \mid \mathbf{x}\rangle},
$$

but also with a direct orthonormal frame whose choice will be specified later on. Inside this frame, every point $\mathbf{x} \in \mathbb{R}^{n}$ will be written in the form $\left(\mathbf{x}^{\prime}, x_{n}\right)$ so that $\mathbf{x}^{\prime}=\left(x_{1}, \ldots, x_{n-1}\right) \in \mathbb{R}^{n-1}$. In particular, the symbol $\mathbf{0}$ (resp. $\mathbf{0}^{\prime}$ ) refers to the zero vector of $\mathbb{R}^{n}$ (resp. of $\mathbb{R}^{n-1}$ ). First, the uniform ball condition is defined as follows.

Definition 1.1. Consider a real number $\varepsilon>0$ and let $\Omega$ be an open subset of $\mathbb{R}^{n}$. Its closure is referred as $\bar{\Omega}$ and its boundary is marked $\partial \Omega=\bar{\Omega} \backslash \Omega$. We say that $\Omega$ satisfies the $\varepsilon$-ball condition if there exists for every point $\mathbf{x} \in \partial \Omega$, a certain unit vector $\mathbf{d}_{\mathbf{x}}$ of $\mathbb{R}^{n}$ such that:

$$
\left\{\begin{array}{l}
B_{\varepsilon}\left(\mathbf{x}-\varepsilon \mathbf{d}_{\mathbf{x}}\right) \subseteq \Omega \\
B_{\varepsilon}\left(\mathbf{x}+\varepsilon \mathbf{d}_{\mathbf{x}}\right) \subseteq \mathbb{R}^{n} \backslash \bar{\Omega}
\end{array}\right.
$$

where $B_{\varepsilon}\left(\mathbf{x} \pm \varepsilon \mathbf{d}_{\mathbf{x}}\right)=\left\{\mathbf{y} \in \mathbb{R}^{n}, \quad\left\|\mathbf{y}-\mathbf{x} \mp \varepsilon \mathbf{d}_{\mathbf{x}}\right\|<\varepsilon\right\}$ is the open ball of $\mathbb{R}^{n}$ centred at $\mathbf{x} \pm \varepsilon \mathbf{d}_{\mathbf{x}}$ and of radius $\varepsilon$. 
Then, some of the notation introduced in [12] by Federer are recalled. For every non-empty subset $A$ of $\mathbb{R}^{n}$, the following map is well defined and 1-Lipschitz continuous:

$$
\begin{aligned}
d_{A}: \mathbb{R}^{n} & \longrightarrow[0,+\infty[ \\
\mathbf{x} & \longmapsto d_{A}(\mathbf{x})=\inf _{\mathbf{a} \in A}\|\mathbf{x}-\mathbf{a}\|
\end{aligned}
$$

Furthermore, we introduce the following set:

$$
\operatorname{Unp}(A)=\left\{\mathbf{x} \in \mathbb{R}^{n} \mid \exists ! \mathbf{a} \in A, \quad\|\mathbf{x}-\mathbf{a}\|=d_{A}(\mathbf{x})\right\}
$$

It contains all the points of $\mathbb{R}^{n}$ having a unique projection on $A$, that is the maximal domain on which the following map is well defined:

$$
\begin{aligned}
p_{A}: \operatorname{Unp}(A) & \longrightarrow A \\
\mathbf{x} & \longmapsto p_{A}(\mathbf{x}),
\end{aligned}
$$

where $p_{A}(\mathbf{x})$ is the unique point of $A$ such that $\left\|p_{A}(\mathbf{x})-\mathbf{x}\right\|=d_{A}(\mathbf{x})$. We can also notice that $A \subseteq \operatorname{Unp}(A)$ thus in particular $\operatorname{Unp}(A) \neq \emptyset$. We can now express what is a set of positive reach.

Definition 1.2. Consider a non-empty subset $A$ of $\mathbb{R}^{n}$. First, for every point $\mathbf{a} \in A$, we set the notation:

$$
\operatorname{Reach}(A, \mathbf{a})=\sup \left\{r>0, \quad B_{r}(\mathbf{a}) \subseteq \operatorname{Unp}(A)\right\}
$$

with the conventions $\sup \emptyset=0$ and also sup $X=+\infty$ if the set $X$ is not empty and not bounded from above. Then, we define the reach of the set $A$ by the following quantity:

$$
\operatorname{Reach}(A)=\inf _{\mathbf{a} \in A} \operatorname{Reach}(A, \mathbf{a})
$$

and $\operatorname{Reach}(A)=+\infty$ if $\operatorname{Reach}(A, \mathbf{a})=+\infty$ for all $\mathbf{a} \in A$. Finally, we say $A$ has a positive $\operatorname{reach}$ if $\operatorname{Reach}(A)>0$.

Remark 1.3. According to Definition 1.2, the reach of a subset of $\mathbb{R}^{n}$ is well defined as soon as it is not empty. Consequently, when considering the reach associated to the boundary of an open subset $\Omega$ of $\mathbb{R}^{n}$, we will have to ensure $\partial \Omega \neq \emptyset$ and to do so, we will always assume $\Omega$ is not empty and different from $\mathbb{R}^{n}$. Indeed, if $\partial \Omega=\emptyset$, then $\bar{\Omega}=\Omega \cup \partial \Omega=\Omega$ thus $\Omega=\emptyset$ or $\Omega=\mathbb{R}^{n}$ because these are the only open and closed subsets of $\mathbb{R}^{n}$.

Finally, we recall here the definition of the uniform cone property introduced by Chenais in [10] and also the notion of a $C^{1,1}$-hypersurface of $\mathbb{R}^{n}$ in terms of local graph.

Definition 1.4. Consider a real number $\alpha \in] 0, \frac{\pi}{2}\left[\right.$ and let $\Omega$ be an open subset of $\mathbb{R}^{n}$. We say that $\Omega$ satisfies the $\alpha$-cone condition if there exists for every point $\mathbf{x} \in \partial \Omega$, a certain unit vector $\xi_{\mathbf{x}}$ of $\mathbb{R}^{n}$ such that:

$$
\forall \mathbf{y} \in B_{\alpha}(\mathbf{x}) \cap \Omega, \quad C_{\alpha}\left(\mathbf{y}, \xi_{\mathbf{x}}\right) \subseteq \Omega
$$

with $C_{\alpha}\left(\mathbf{y}, \xi_{\mathbf{x}}\right)=\left\{\mathbf{z} \in B_{\alpha}(\mathbf{y}),\|\mathbf{z}-\mathbf{y}\| \cos \alpha<\left\langle\mathbf{z}-\mathbf{y} \mid \xi_{\mathbf{x}}\right\rangle\right\}$ the open cone of corner $\mathbf{y}$, direction $\xi_{\mathbf{x}}$, and span $\alpha$. Definition 1.5. Consider a subset $\mathcal{S}$ of $\mathbb{R}^{n}$. We say that $\mathcal{S}$ is a $C^{1,1}$-hypersurface if there exists for every $\mathrm{x}_{\mathbf{0}} \in \mathcal{S}$, a direct orthonormal frame centred at $\mathbf{x}_{\mathbf{0}}$ such that in this local frame, there exists a map $\left.\varphi: D_{r}\left(\mathbf{0}^{\prime}\right) \rightarrow\right]-a, a[$ continuously differentiable with $a>0$, such that $\varphi$ and its gradient $\nabla \varphi$ are $L$-Lipschitz continuous with $L>0$, satisfying $\varphi\left(\mathbf{0}^{\prime}\right)=0, \nabla \varphi\left(\mathbf{0}^{\prime}\right)=\mathbf{0}^{\prime}$, and also:

$$
\left\{\begin{array}{l}
\partial \Omega \cap\left(D_{r}\left(\mathbf{0}^{\prime}\right) \times\right]-a, a[)=\left\{\left(\mathbf{x}^{\prime}, \varphi\left(\mathbf{x}^{\prime}\right)\right), \mathbf{x}^{\prime} \in D_{r}\left(\mathbf{0}^{\prime}\right)\right\} \\
\Omega \cap\left(D_{r}\left(\mathbf{0}^{\prime}\right) \times\right]-a, a[)=\left\{\left(\mathbf{x}^{\prime}, x_{n}\right), \quad \mathbf{x}^{\prime} \in D_{r}\left(\mathbf{0}^{\prime}\right) \text { and }-a<x_{n}<\varphi\left(\mathbf{x}^{\prime}\right)\right\}
\end{array}\right.
$$

where $D_{r}\left(\mathbf{0}^{\prime}\right)=\left\{\mathbf{x}^{\prime} \in \mathbb{R}^{n-1}, \quad\left\|\mathbf{x}^{\prime}\right\|<r\right\}$ is the open ball of $\mathbb{R}^{n-1}$ centred at the origin $\mathbf{0}^{\prime}$ and of radius $r>0$. 


\subsection{A characterization in terms of positive reach}

Theorem 1.6. Consider a non-empty open subset $\Omega$ of $\mathbb{R}^{n}$ different from $\mathbb{R}^{n}$. Then, the following implications are true:

(i) if there exists a real number $\varepsilon>0$ such that $\Omega$ satisfies the $\varepsilon$-ball condition as in Definition 1.1 , then $\partial \Omega$ has a positive reach in the sense of Definition 1.2 and we have $\operatorname{Reach}(\partial \Omega) \geqslant \varepsilon$;

(ii) if $\partial \Omega$ has a positive reach, then $\Omega$ satisfies the $\varepsilon$-ball condition for every real number $\varepsilon \in] 0, \operatorname{Reach}(\partial \Omega)[$, and moreover, if $\partial \Omega$ has a finite positive reach, then $\Omega$ also satisfies the $\operatorname{Reach}(\partial \Omega)$-ball condition.

Corollary 1.7. If $\Omega$ is a non-empty open subset of $\mathbb{R}^{n}$ different from $\mathbb{R}^{n}$, then we have the characterization:

$$
\operatorname{Reach}(\partial \Omega)=\sup \{\varepsilon>0 \mid \Omega \text { satisfies the } \varepsilon \text {-ball condition as in Definition } 1.1\} \text {, }
$$

with the conventions $\sup \emptyset=0$ and also $\sup X=+\infty$ if the set $X$ is not empty and not bounded from above. Moreover, this supremum becomes a maximum as soon as it is not zero and finite. Finally, $\operatorname{Reach}(\partial \Omega)=+\infty$ if and only if $\partial \Omega$ is an affine hyperplane of $\mathbb{R}^{n}$.

\subsection{A characterization in terms of $C^{1,1}$-regularity}

Theorem 1.8. Let $\Omega$ be a non-empty open subset of $\mathbb{R}^{n}$ different from $\mathbb{R}^{n}$. If there exists a real number $\varepsilon>0$ such that $\Omega$ satisfies the $\varepsilon$-ball condition as in Definition 1.1, then its boundary $\partial \Omega$ is a $C^{1,1}$-hypersurface in the sense of Definition 1.5 where $a=\varepsilon$, and the constants $L, r$ depend only on $\varepsilon$. Moreover, we have the following properties:

(i) $\Omega$ satisfies the $f^{-1}(\varepsilon)$-cone property as in Definition 1.4, where we set $\left.f: \alpha \in\right] 0, \frac{\pi}{2}\left[\mapsto \frac{2 \alpha}{\cos \alpha} \in\right] 0,+\infty[$;

(ii) the unit vector $\mathbf{d}_{\mathbf{x}}$ of Definition 1.1 is the outer normal to the surface $\partial \Omega$ at the point $\mathbf{x}$;

(iii) the Gauss map $\mathbf{d}: \mathbf{x} \in \partial \Omega \longmapsto \mathbf{d}_{\mathbf{x}}$ is well defined and $\frac{1}{\varepsilon}$-Lipschitz continuous.

Theorem 1.9. Consider a non-empty compact $C^{1,1}$-hypersurface $\mathcal{S}$ of $\mathbb{R}^{n}$ in the sense of Definition 1.5. Then, $\mathcal{S}$ is orientable and there exists a unique bounded open subset $\Omega$ of $\mathbb{R}^{n}$ such that $\partial \Omega=\mathcal{S}$. Moreover, there exists a real number $\varepsilon>0$ such that $\Omega$ satisfies the $\varepsilon$-ball condition as in Definition 1.1.

\section{Sets of POSitive REACH AND the Uniform BALl CONDition}

Throughout this section, $\Omega$ refers to any non-empty open subset of $\mathbb{R}^{n}$ different from $\mathbb{R}^{n}$. Hence, its boundary $\partial \Omega$ is not empty and Reach $(\partial \Omega$ ) is well defined (cf. Remark 1.3). First, we establish some useful properties that were mentioned in Federer's paper [12] and then, we show that the two assertions of Theorem 1.6 hold.

\subsection{Positive reach implies uniform ball condition}

Lemma 2.1. For every point $\mathbf{x} \in \partial \Omega$, we have: $\operatorname{Reach}(\partial \Omega, \mathbf{x})=\min \left(\operatorname{Reach}(\bar{\Omega}, \mathbf{x}), \operatorname{Reach}\left(\mathbb{R}^{n} \backslash \Omega, \mathbf{x}\right)\right)$.

Proof. We only sketch the proof. Observe that $d_{\partial \Omega}(\mathbf{x})=\max \left(d_{\bar{\Omega}}(\mathbf{x}), d_{\mathbb{R}^{n} \backslash \Omega}(\mathbf{x})\right)$ for any $\mathbf{x} \in \mathbb{R}^{n}$ in order to get $\operatorname{Unp}(\partial \Omega)=\operatorname{Unp}(\bar{\Omega}) \cap \operatorname{Unp}\left(\mathbb{R}^{n} \backslash \Omega\right)$ and the equality of the statement follows from definitions.

Proposition 2.2 (Federer [12] Theorem 4.8 (6) page 434). Consider a non-empty closed subset $A$ of $\mathbb{R}^{n}$, a point $\mathbf{x} \in A$, and a vector $\mathbf{v}$ of $\mathbb{R}^{n}$. If the set $\left\{t>0, \mathbf{x}+t \mathbf{v} \in \operatorname{Unp}(A)\right.$ and $\left.p_{A}(\mathbf{x}+t \mathbf{v})=\mathbf{x}\right\}$ is not empty and bounded from above, then its supremum $\tau$ is well defined and $\mathbf{x}+\tau \mathbf{v}$ cannot belong to the interior of $\operatorname{Unp}(A)$.

Proof. We refer to [12] for a proof based on Peano's existence theorem for solutions of differential equations. 
Corollary 2.3. For every point $\mathbf{x} \in \partial \Omega$ satisfying $\operatorname{Reach}(\partial \Omega, \mathbf{x})>0$, there exists two points $\mathbf{y} \in \operatorname{Unp}(\bar{\Omega}) \backslash\{\mathbf{x}\}$ and $\tilde{\mathbf{y}} \in \operatorname{Unp}\left(\mathbb{R}^{n} \backslash \Omega\right) \backslash\{\mathbf{x}\}$ such that $p_{\bar{\Omega}}(\mathbf{y})=p_{\mathbb{R}^{n} \backslash \Omega}(\tilde{\mathbf{y}})=\mathbf{x}$.

Proof. Consider any $\mathbf{x} \in \partial \Omega$ that satisfies $\operatorname{Reach}(\partial \Omega, \mathbf{x})>0$. From Lemma 2.1, there exists a real number $r>0$ such that $\overline{B_{r}(\mathbf{x})} \subseteq \operatorname{Unp}(\bar{\Omega})$. We consider a sequence $\left(\mathbf{x}_{i}\right)_{i \in \mathbb{N}}$ of elements of $B_{\frac{r}{2}}(\mathbf{x}) \backslash \bar{\Omega}$ converging to $\mathbf{x}$. We set:

$$
\forall i \in \mathbb{N}, \forall t \in \mathbb{R}, \quad \mathbf{z}_{i}(t)=p_{\bar{\Omega}}\left(\mathbf{x}_{i}\right)+t \frac{\mathbf{x}_{i}-p_{\bar{\Omega}}\left(\mathbf{x}_{i}\right)}{\left\|\mathbf{x}_{i}-p_{\bar{\Omega}}\left(\mathbf{x}_{i}\right)\right\|} \quad \text { and } \quad t_{i}=\frac{r}{2}+d_{\bar{\Omega}}\left(\mathbf{x}_{i}\right)
$$

which is well defined since $\mathbf{x}_{i} \in \operatorname{Unp}(\bar{\Omega})$. First, observe that $\mathbf{z}_{i}(t) \in \overline{B_{\frac{r}{2}}\left(\mathbf{x}_{i}\right)} \subseteq B_{r}(\mathbf{x}) \subseteq \operatorname{Unp}(\bar{\Omega})$ for any $t \in\left[0, t_{i}\right]$. Then, using Federer's result recalled in Proposition 2.2, one can prove by contradiction that:

$$
\forall t \in\left[0, t_{i}\right], \quad p_{\bar{\Omega}}\left(\mathbf{z}_{i}(t)\right)=p_{\bar{\Omega}}\left(\mathbf{x}_{i}\right) .
$$

Finally, the sequence $\mathbf{y}_{i}=\mathbf{z}_{i}\left(t_{i}\right)$ satisfies $\left\|\mathbf{y}_{i}-\mathbf{x}_{i}\right\|=\frac{r}{2}$ and also $p_{\bar{\Omega}}\left(\mathbf{y}_{i}\right)=p_{\bar{\Omega}}\left(\mathbf{x}_{i}\right)$. Moreover, since it is bounded, $\left(\mathbf{y}_{i}\right)_{i \in \mathbb{N}}$ is converging, up to a subsequence, to a certain point $\mathbf{y} \in \overline{B_{r}(\mathbf{x})} \subseteq \operatorname{Unp}(\bar{\Omega})$. Using the continuity of $p_{\bar{\Omega}}$ (cf. [12] Theorem 4.8 (4) page 434), we get $\mathbf{y} \in \operatorname{Unp}(\bar{\Omega}) \backslash\{\mathbf{x}\}$ and $p_{\bar{\Omega}}(\mathbf{y})=p_{\bar{\Omega}}(\mathbf{x})=\mathbf{x}$. To conclude, the same arguments work when replacing $\bar{\Omega}$ by the closed set $\mathbb{R}^{n} \backslash \Omega$. Hence, the result of the statement is established.

Proposition 2.4 (Point (ii) of Theorem 1.6). If $\partial \Omega$ is a set of positive reach, then $\Omega$ satisfies the $\varepsilon$-ball condition for any $\varepsilon \in] 0, \operatorname{Reach}(\partial \Omega)[$. Moreover, if $0<\operatorname{Reach}(\partial \Omega)<+\infty$, then it is also true for $\varepsilon=\operatorname{Reach}(\partial \Omega)$.

Proof. As $\Omega \notin\left\{\emptyset, \mathbb{R}^{n}\right\}, \partial \Omega \neq \emptyset$ so its reach is well defined. Assume $\operatorname{Reach}(\partial \Omega)>0$ and choose $\left.\varepsilon \in\right] 0, \operatorname{Reach}(\partial \Omega)[$. Consider $\mathbf{x} \in \partial \Omega$. From Corollary 2.3, there exists $\mathbf{y} \in \operatorname{Unp}(\bar{\Omega}) \backslash\{\mathbf{x}\}$ such that $p_{\bar{\Omega}}(\mathbf{y})=\mathbf{x}$ so we set $\mathbf{d}_{\mathbf{x}}=\frac{\mathbf{x}-\mathbf{y}}{\|\mathbf{x}-\mathbf{y}\|}$. From Lemma 2.1, we get $\mathbf{x}+[0, \varepsilon] \mathbf{d}_{\mathbf{x}} \subseteq \operatorname{Unp}(\bar{\Omega})$, then use Proposition 2.2 again to prove by contradiction that:

$$
\forall t \in[0, \varepsilon], \quad p_{\bar{\Omega}}\left(\mathbf{x}+\varepsilon \mathbf{d}_{\mathbf{x}}\right)=\mathbf{x} .
$$

In particular, we have $\left\|\mathbf{z}-\left(\mathbf{x}+\varepsilon \mathbf{d}_{\mathbf{x}}\right)\right\|>\varepsilon$ for every point $\mathbf{z} \in \bar{\Omega} \backslash\{\mathbf{x}\}$ from which we deduce that:

$$
\bar{\Omega} \subseteq\{\mathbf{x}\} \cup\left(\mathbb{R}^{n} \backslash \overline{B_{\varepsilon}\left(\mathbf{x}+\varepsilon \mathbf{d}_{\mathbf{x}}\right)}\right) \Longleftrightarrow \overline{B_{\varepsilon}\left(\mathbf{x}+\varepsilon \mathbf{d}_{\mathbf{x}}\right)} \backslash\{\mathbf{x}\} \subseteq \mathbb{R}^{n} \backslash \bar{\Omega} .
$$

Similarly, there exists a unit vector $\xi_{\mathbf{x}}$ such that $\overline{B_{\varepsilon}\left(\mathbf{x}+\varepsilon \xi_{\mathbf{x}}\right)} \backslash\{\mathbf{x}\} \subseteq \Omega$. Since $\overline{B_{\varepsilon}\left(\mathbf{x}+\varepsilon \xi_{\mathbf{x}}\right)} \cap \overline{B_{\varepsilon}\left(\mathbf{x}+\varepsilon \mathbf{d}_{\mathbf{x}}\right)}=\{\mathbf{x}\}$, we obtain $\mathbf{d}_{\mathbf{x}}=-\xi_{\mathbf{x}}$. To conclude, if $\operatorname{Reach}(\partial \Omega)<+\infty$, then observe that $B_{\operatorname{Reach}(\partial \Omega)}\left(\mathbf{x} \pm \operatorname{Reach}(\partial \Omega) \mathbf{d}_{\mathbf{x}}\right)$ is equal to $\bigcup_{0<\varepsilon<\operatorname{Reach}(\partial \Omega)} \overline{B_{\varepsilon}\left(\mathbf{x} \pm \varepsilon \mathbf{d}_{\mathbf{x}}\right)} \backslash\{\mathbf{x}\}$ in order to check that $\Omega$ also satisfies the Reach $(\partial \Omega)$-ball condition.

\subsection{Uniform ball condition implies positive reach}

Proposition 2.5. Assume that the open set $\Omega$ satisfies the $\varepsilon$-ball condition for a certain real number $\varepsilon>0$. Then, for every $(\mathbf{x}, \mathbf{y}) \in \partial \Omega \times \partial \Omega$, the following inequality holds: $\left\|\mathbf{d}_{\mathbf{x}}-\mathbf{d}_{\mathbf{y}}\right\| \leqslant \frac{1}{\varepsilon}\|\mathbf{x}-\mathbf{y}\|$. In particular, if $\mathbf{x}=\mathbf{y}$, then $\mathbf{d}_{\mathbf{x}}=\mathbf{d}_{\mathbf{y}}$ which ensures uniqueness of the unit vector $\mathbf{d}_{\mathbf{x}}$ in Definition 1.1 associated to each point $\mathbf{x} \in \partial \Omega$. In other words, the map $\mathbf{d}: \mathbf{x} \in \partial \Omega \mapsto \mathbf{d}_{\mathbf{x}}$ is well defined and $\frac{1}{\varepsilon}$-Lipschitz continuous.

Proof. Consider $\varepsilon>0$ and assume that $\Omega$ satisfies the $\varepsilon$-ball condition. Since $\Omega \notin\left\{\emptyset, \mathbb{R}^{n}\right\}, \partial \Omega$ is not empty so choose $(\mathbf{x}, \mathbf{y}) \in \partial \Omega \times \partial \Omega$. First, the balls contained in $\Omega$ cannot intersect those inside $\mathbb{R}^{n} \backslash \bar{\Omega}$. Therefore, we have: $B_{\varepsilon}\left(\mathbf{x} \pm \varepsilon \mathbf{d}_{\mathbf{x}}\right) \cap B_{\varepsilon}\left(\mathbf{y} \mp \varepsilon \mathbf{d}_{\mathbf{y}}\right)=\emptyset \Longleftrightarrow\left\|\mathbf{x}-\mathbf{y} \pm \varepsilon\left(\mathbf{d}_{\mathbf{x}}+\mathbf{d}_{\mathbf{y}}\right)\right\| \geqslant 2 \varepsilon$. Indeed, two open balls are disconnected if and only if the distance between their centre is at least the sum of their radius. Then, squaring these two inequalities and summing them, one obtains the result of the statement: $\|\mathbf{x}-\mathbf{y}\|^{2} \geqslant 2 \varepsilon^{2}-2 \varepsilon^{2}\left\langle\mathbf{d}_{\mathbf{x}} \mid \mathbf{d}_{\mathbf{y}}\right\rangle=\varepsilon^{2}\left\|\mathbf{d}_{\mathbf{x}}-\mathbf{d}_{\mathbf{y}}\right\|^{2}$.

Proposition 2.6 (Point (i) of Theorem 1.6). If $\Omega$ satisfies the $\varepsilon$-ball condition, then $\operatorname{Reach}(\partial \Omega) \geqslant \varepsilon>0$. 
Proof. Consider $\varepsilon>0$ and assume that $\Omega$ satisfies the $\varepsilon$-ball condition. Since $\Omega \notin\left\{\emptyset, \mathbb{R}^{n}\right\}$, $\partial \Omega$ is not empty so choose $\mathbf{x} \in \partial \Omega$ and let us prove $B_{\varepsilon}(\mathbf{x}) \subseteq \operatorname{Unp}(\partial \Omega)$. First, we consider any $\mathbf{y} \in B_{\varepsilon}(\mathbf{x}) \cap \Omega$. Since $\partial \Omega$ is closed, there exists $\mathbf{z} \in \partial \Omega$ such that $d_{\partial \Omega}(\mathbf{y})=\|\mathbf{z}-\mathbf{y}\|$. Moreover, we obtain from the $\varepsilon$-ball condition and $\mathbf{y} \in \Omega$ :

$$
\left\{\begin{array}{l}
B_{\varepsilon}\left(\mathbf{z}+\varepsilon \mathbf{d}_{\mathbf{z}}\right) \subseteq \mathbb{R}^{n} \backslash \bar{\Omega} \\
B_{d_{\partial \Omega}(\mathbf{y})}(\mathbf{y}) \subseteq \Omega
\end{array} \quad \Longrightarrow \quad B_{\varepsilon}\left(\mathbf{z}+\varepsilon \mathbf{d}_{\mathbf{z}}\right) \cap B_{d_{\partial \Omega}(\mathbf{y})}(\mathbf{y})=\emptyset .\right.
$$

Hence, we deduce that $\mathbf{y}=\mathbf{z}-d_{\partial \Omega}(\mathbf{y}) \mathbf{d}_{\mathbf{z}}$. Then, we show that such a $\mathbf{z}$ is unique. Considering another projection $\tilde{\mathbf{z}}$ of $\mathbf{y}$ on $\partial \Omega$, we get from the foregoing: $\mathbf{y}=\mathbf{z}-d_{\partial \Omega}(\mathbf{y}) \mathbf{d}_{\mathbf{z}}=\tilde{\mathbf{z}}-d_{\partial \Omega}(\mathbf{y}) \mathbf{d}_{\tilde{\mathbf{z}}}$. Using Proposition 2.5, we have:

$$
\left\|\mathbf{d}_{\mathbf{z}}-\mathbf{d}_{\tilde{\mathbf{z}}}\right\| \leqslant \frac{1}{\varepsilon}\|\mathbf{z}-\tilde{\mathbf{z}}\|=\frac{d_{\partial \Omega}(\mathbf{y})}{\varepsilon}\left\|\mathbf{d}_{\mathbf{z}}-\mathbf{d}_{\tilde{\mathbf{z}}}\right\| .
$$

Since $d_{\partial \Omega}(\mathbf{y}) \leqslant\|\mathbf{x}-\mathbf{y}\|<\varepsilon$, the above inequality can only hold if $\left\|\mathbf{d}_{\mathbf{z}}-\mathbf{d}_{\tilde{\mathbf{z}}}\right\|=0$ i.e. $\mathbf{z}=\tilde{\mathbf{z}}$. Hence, we obtain $B_{\varepsilon}(\mathbf{x}) \cap \Omega \subseteq \operatorname{Unp}(\partial \Omega)$ and similarly, one can prove that $B_{\varepsilon}(\mathbf{x}) \cap\left(\mathbb{R}^{n} \backslash \bar{\Omega}\right) \subseteq \operatorname{Unp}(\partial \Omega)$. Since $\partial \Omega \subseteq \operatorname{Unp}(\partial \Omega)$, we finally get $B_{\varepsilon}(\mathbf{x}) \subseteq \operatorname{Unp}(\partial \Omega)$. To conclude, we have Reach $(\partial \Omega, \mathbf{x}) \geqslant \varepsilon$ for every $\mathbf{x} \in \partial \Omega$ i.e. Reach $(\partial \Omega) \geqslant \varepsilon$.

Proposition 2.7. Assume that the open set $\Omega$ satisfies the $\varepsilon$-ball condition for a certain a real number $\varepsilon>0$. Then, for every $(\mathbf{a}, \mathbf{x}) \in \partial \Omega \times \partial \Omega$, the following global inequality holds: $\left|\left\langle\mathbf{x}-\mathbf{a} \mid \mathbf{d}_{\mathbf{a}}\right\rangle\right| \leqslant \frac{1}{2 \varepsilon}\|\mathbf{x}-\mathbf{a}\|^{2}$. Moreover, if we introduce the vector $(\mathbf{x}-\mathbf{a})^{\prime}=(\mathbf{x}-\mathbf{a})-\left\langle\mathbf{x}-\mathbf{a} \mid \mathbf{d}_{\mathbf{a}}\right\rangle \mathbf{d}_{\mathbf{a}}$ and assume $\left\|(\mathbf{x}-\mathbf{a})^{\prime}\right\|<\varepsilon$ and also $\left|\left\langle\mathbf{x}-\mathbf{a} \mid \mathbf{d}_{\mathbf{a}}\right\rangle\right|<\varepsilon$, then the following local inequality holds: $\frac{1}{2 \varepsilon}\|\mathbf{x}-\mathbf{a}\|^{2} \leqslant \varepsilon-\sqrt{\varepsilon^{2}-\left\|(\mathbf{x}-\mathbf{a})^{\prime}\right\|^{2}}$.

Proof. Consider $\varepsilon>0$ and assume that $\Omega$ satisfies the $\varepsilon$-ball condition. Since $\Omega \notin\left\{\emptyset, \mathbb{R}^{n}\right\}, \partial \Omega$ is not empty so choose $(\mathbf{a}, \mathbf{x}) \in \partial \Omega \times \partial \Omega$. Since the point $\mathbf{x}$ cannot belong neither to $B_{\varepsilon}\left(\mathbf{a}-\varepsilon \mathbf{d}_{\mathbf{a}}\right) \subseteq \Omega$ nor to $B_{\varepsilon}\left(\mathbf{a}+\varepsilon \mathbf{d}_{\mathbf{a}}\right) \subseteq \mathbb{R}^{n} \backslash \bar{\Omega}$, we have $\left\|\mathbf{x}-\mathbf{a} \mp \varepsilon \mathbf{d}_{\mathbf{a}}\right\| \geqslant \varepsilon$. Squaring the two inequalities, we obtain the global inequality of the statement:

$$
\|\mathbf{x}-\mathbf{a}\|^{2} \geqslant 2 \varepsilon\left|\left\langle\mathbf{x}-\mathbf{a} \mid \mathbf{d}_{\mathbf{a}}\right\rangle\right| \Longleftrightarrow\left|\left\langle\mathbf{x}-\mathbf{a} \mid \mathbf{d}_{\mathbf{a}}\right\rangle\right|^{2}-2 \varepsilon\left|\left\langle\mathbf{x}-\mathbf{a} \mid \mathbf{d}_{\mathbf{a}}\right\rangle\right|+\left\|(\mathbf{x}-\mathbf{a})^{\prime}\right\|^{2} \geqslant 0 .
$$

It is a second-order polynomial inequality whose discriminant is assumed to be positive: $\Delta^{\prime}=\varepsilon^{2}-\left\|(\mathbf{x}-\mathbf{a})^{\prime}\right\|^{2}>0$. Hence, it will be fulfilled if the unknown is not located between the two roots: either $\left|\left\langle\mathbf{x}-\mathbf{a} \mid \mathbf{d}_{\mathbf{a}}\right\rangle\right| \leqslant \varepsilon-\sqrt{\Delta^{\prime}}$ or $\left|\left\langle\mathbf{x}-\mathbf{a} \mid \mathbf{d}_{\mathbf{a}}\right\rangle\right| \geqslant \varepsilon+\sqrt{\Delta^{\prime}}$. Assume $\left|\left\langle\mathbf{x}-\mathbf{a} \mid \mathbf{d}_{\mathbf{a}}\right\rangle\right|<\varepsilon$ and the last case cannot hold. Squaring the remaining relation, we get the local inequality: $\|\mathbf{x}-\mathbf{a}\|^{2}=\left|\left\langle\mathbf{x}-\mathbf{a} \mid \mathbf{d}_{\mathbf{a}}\right\rangle\right|^{2}+\left\|(\mathbf{x}-\mathbf{a})^{\prime}\right\|^{2} \leqslant 2 \varepsilon^{2}-2 \varepsilon \sqrt{\varepsilon^{2}-\left\|(\mathbf{x}-\mathbf{a})^{\prime}\right\|^{2}}$.

\section{The Uniform Ball CONDition AND the COMPACt $C^{1,1}$-HyPERSURFACES}

In this section, we prove Theorem 1.8 and Theorem 1.9. First, we show that $\partial \Omega$ can be considered locally as the graph of a function whose $C^{1,1}$-regularity is then established. Finally, we demonstrate that the converse statement holds in the compact case. Hence, it is the optimal regularity we can expect from the $\varepsilon$-ball property.

\subsection{A local parametrization of $\partial \Omega$}

Set $\varepsilon>0$ and assume that the open set $\Omega$ satisfies the $\varepsilon$-ball condition. Since $\Omega \notin\left\{\emptyset, \mathbb{R}^{n}\right\}, \partial \Omega$ is not empty so consider $\mathbf{x}_{\mathbf{0}} \in \partial \Omega$. Its vector $\mathbf{d}_{\mathbf{x}_{0}}$ is unique from Proposition 2.5. Choose a basis $\mathcal{B}_{\mathbf{x}_{0}}$ of the hyperplane $\mathbf{d}_{\mathbf{x}_{0}} \perp$ such that $\left(\mathbf{x}_{\mathbf{0}}, \mathcal{B}_{\mathbf{x}_{0}}, \mathbf{d}_{\mathbf{x}_{0}}\right)$ is a direct orthonormal frame. Hence, every $\mathbf{x} \in \mathbb{R}^{n}$ is of the form $\left(\mathbf{x}^{\prime}, x_{n}\right)$ such that $\mathbf{x}^{\prime}=\left(x_{1}, \ldots, x_{n-1}\right)$. The zero vector $\mathbf{0}$ is identified with $\mathbf{x}_{\mathbf{0}}$ so we have $B_{\varepsilon}\left(\mathbf{0}^{\prime},-\varepsilon\right) \subseteq \Omega$ and $B_{\varepsilon}\left(\mathbf{0}^{\prime}, \varepsilon\right) \subseteq \mathbb{R}^{n} \backslash \bar{\Omega}$.

Proposition 3.1. Set $D_{\varepsilon}\left(\mathbf{0}^{\prime}\right)=\left\{\mathbf{x}^{\prime} \in \mathbb{R}^{n-1},\left\|\mathbf{x}^{\prime}\right\|<\varepsilon\right\}$ the open ball of $\mathbb{R}^{n-1}$ of radius $\varepsilon$ centred at $\mathbf{0}^{\prime}$ and also:

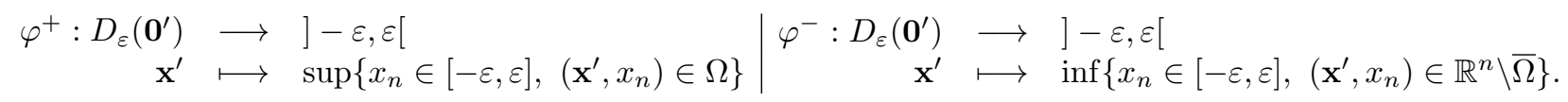

Then, $\varphi^{ \pm}$are two well-defined maps. Moreover, for every $\mathbf{x}^{\prime} \in D_{\varepsilon}\left(\mathbf{0}^{\prime}\right)$, introducing the points $\mathbf{x}^{ \pm}=\left(\mathbf{x}^{\prime}, \varphi^{ \pm}\left(\mathbf{x}^{\prime}\right)\right)$, we have $\mathbf{x}^{ \pm} \in \partial \Omega$ and the following inequalities hold: $\left|\varphi^{ \pm}\left(\mathbf{x}^{\prime}\right)\right| \leqslant \frac{1}{2 \varepsilon}\left\|\mathbf{x}^{ \pm}-\mathbf{x}_{\mathbf{0}}\right\|^{2} \leqslant \varepsilon-\sqrt{\varepsilon^{2}-\left\|\mathbf{x}^{\prime}\right\|}$. 
Proof. Set $\mathbf{x}^{\prime} \in D_{\varepsilon}\left(\mathbf{0}^{\prime}\right)$ and consider the continuous map $g: t \in[-\varepsilon, \varepsilon] \mapsto\left(\mathbf{x}^{\prime}, t\right)$. Since $-\varepsilon \in g^{-1}(\Omega) \subseteq[-\varepsilon, \varepsilon]$, we can set $\varphi^{+}\left(\mathbf{x}^{\prime}\right)=\sup g^{-1}(\Omega)$ but $g^{-1}(\Omega)$ is open so we get $\varphi^{+}\left(\mathbf{x}^{\prime}\right) \notin g^{-1}(\Omega)$ i.e. $\mathbf{x}^{+} \in \bar{\Omega} \backslash \Omega=\partial \Omega$. Similarly, the map $\varphi^{-}$is well defined and $\mathbf{x}^{-} \in \partial \Omega$. Finally, apply Proposition 2.7 to the points $\mathbf{a}=\mathbf{x}_{\mathbf{0}}$ and $\mathbf{x}=\mathbf{x}^{ \pm}$.

Lemma 3.2. Set $r=\frac{\sqrt{3}}{2} \varepsilon$. Consider $\mathbf{x}^{\prime} \in D_{r}\left(\mathbf{0}^{\prime}\right)$. Assume there exists $\left.x_{n} \in\right]-\varepsilon, \varepsilon\left[\right.$ such that $\mathbf{x}=\left(\mathbf{x}^{\prime}, x_{n}\right) \in \partial \Omega$ and also another real number $\tilde{x}_{n}$ satisfying the inequality $\left|\tilde{x}_{n}\right| \leqslant \varepsilon-\sqrt{\varepsilon^{2}-\left\|\mathbf{x}^{\prime}\right\|^{2}}$. Then, introduce $\tilde{\mathbf{x}}=\left(\mathbf{x}^{\prime}, \tilde{x}_{n}\right)$ and the following two implications hold: $\left(\tilde{x}_{n}<x_{n} \Longrightarrow \tilde{\mathbf{x}} \in \Omega\right)$ and $\left(\tilde{x}_{n}>x_{n} \Longrightarrow \tilde{\mathbf{x}} \in \mathbb{R}^{n} \backslash \bar{\Omega}\right)$.

Proof. Consider any $\mathbf{x}^{\prime} \in D_{r}\left(\mathbf{0}^{\prime}\right)$. Since $\mathbf{x}-\tilde{\mathbf{x}}=\left(x_{n}-\tilde{x}_{n}\right) \mathbf{d}_{\mathbf{x}_{0}}$, if we assume $\tilde{x}_{n} \gtrless x_{n}$, then we have successively:

$$
\begin{aligned}
\left\|\tilde{\mathbf{x}}-\mathbf{x} \mp \varepsilon \mathbf{d}_{\mathbf{x}}\right\|^{2}-\varepsilon^{2} & =\|\tilde{\mathbf{x}}-\mathbf{x}\|^{2} \mp 2 \varepsilon\left\langle\tilde{\mathbf{x}}-\mathbf{x} \mid \mathbf{d}_{\mathbf{x}}\right\rangle=\left|x_{n}-\tilde{x}_{n}\right|\left(\left|x_{n}-\tilde{x}_{n}\right|+\varepsilon\left\|\mathbf{d}_{\mathbf{x}}-\mathbf{d}_{\mathbf{x}_{\mathbf{0}}}\right\|^{2}-2 \varepsilon\right) \\
& \leqslant\left|x_{n}-\tilde{x}_{n}\right|\left(\left|x_{n}\right|+\left|\tilde{x}_{n}\right|+\frac{1}{\varepsilon}\left\|\mathbf{x}-\mathbf{x}_{\mathbf{0}}\right\|^{2}-2 \varepsilon\right) \leqslant\left|x_{n}-\tilde{x}_{n}\right|\left(2 \varepsilon-4 \sqrt{\varepsilon^{2}-\left\|\mathbf{x}^{\prime}\right\|^{2}}\right)<0 .
\end{aligned}
$$

Indeed, we respectively used the triangle inequality, the one of Proposition 2.5 applied to $\mathbf{x} \in \partial \Omega$ and $\mathbf{y}=\mathbf{x}_{\mathbf{0}}$, the two inequalities of Proposition 2.7 applied to $\mathbf{x} \in \partial \Omega$ and $\mathbf{a}=\mathbf{x}_{\mathbf{0}}$, and also the hypothesis made on $\tilde{x}_{n}$. Hence, we proved that if $\tilde{x}_{n}>x_{n}$, then $\tilde{\mathbf{x}} \in B_{\varepsilon}\left(\mathbf{x}+\varepsilon \mathbf{d}_{\mathbf{x}}\right) \subseteq \mathbb{R}^{n} \backslash \bar{\Omega}$ and if $\tilde{x}_{n}<x_{n}$, then $\tilde{\mathbf{x}} \in B_{\varepsilon}\left(\mathbf{x}-\varepsilon \mathbf{d}_{\mathbf{x}}\right) \subseteq \Omega$.

Proposition 3.3. Set $r=\frac{\sqrt{3}}{2} \varepsilon$. Then, the two maps $\varphi^{ \pm}$defined in Proposition 3.1 coincide on the set $D_{r}\left(\mathbf{0}^{\prime}\right)$ and the function $\varphi$ refers to their common restriction. Moreover, we have $\varphi\left(\mathbf{0}^{\prime}\right)=0$ and the map $\varphi$ satisfies:

$$
\left\{\begin{aligned}
\partial \Omega \cap\left(D_{r}\left(\mathbf{0}^{\prime}\right) \times\right]-\varepsilon, \varepsilon[) & =\left\{\left(\mathbf{x}^{\prime}, \varphi\left(\mathbf{x}^{\prime}\right)\right), \quad \mathbf{x}^{\prime} \in D_{r}\left(\mathbf{0}^{\prime}\right)\right\} \\
\Omega \cap\left(D_{r}\left(\mathbf{0}^{\prime}\right) \times\right]-\varepsilon, \varepsilon[) & =\left\{\left(\mathbf{x}^{\prime}, x_{n}\right), \quad \mathbf{x}^{\prime} \in D_{r}\left(\mathbf{0}^{\prime}\right) \text { and }-\varepsilon<x_{n}<\varphi\left(\mathbf{x}^{\prime}\right)\right\} .
\end{aligned}\right.
$$

Proof. Assume there exists $\mathbf{x}^{\prime} \in D_{r}\left(\mathbf{0}^{\prime}\right)$ such that $\varphi^{-}\left(\mathbf{x}^{\prime}\right) \neq \varphi^{+}\left(\mathbf{x}^{\prime}\right)$. Set $\mathbf{x}=\left(\mathbf{x}^{\prime}, \varphi^{+}\left(\mathbf{x}^{\prime}\right)\right)$ and $\tilde{\mathbf{x}}=\left(\mathbf{x}^{\prime}, \varphi^{-}\left(\mathbf{x}^{\prime}\right)\right)$. From Proposition 3.1, the hypothesis of Lemma 3.2 are satisfied for the two distinct points $\mathbf{x}$ and $\tilde{\mathbf{x}}$. Therefore, we get a contradiction: either $\left(\varphi^{-}\left(\mathbf{x}^{\prime}\right)<\varphi^{+}\left(\mathbf{x}^{\prime}\right) \Rightarrow \tilde{\mathbf{x}} \in \Omega\right)$ or $\left(\varphi^{-}\left(\mathbf{x}^{\prime}\right)>\varphi^{+}\left(\mathbf{x}^{\prime}\right) \Rightarrow \tilde{\mathbf{x}} \in \mathbb{R}^{n} \backslash \bar{\Omega}\right)$ whereas $\tilde{\mathbf{x}} \in \partial \Omega$. Hence, $\varphi^{-}\left(\mathbf{x}^{\prime}\right)=\varphi^{+}\left(\mathbf{x}^{\prime}\right)$ for any $\mathbf{x}^{\prime} \in D_{r}\left(\mathbf{0}^{\prime}\right)$. Now consider $\mathbf{x}^{\prime} \in D_{r}\left(\mathbf{0}^{\prime}\right)$ and $\left.x_{n} \in\right]-\varepsilon, \varepsilon\left[\right.$. We set $\mathbf{x}=\left(\mathbf{x}^{\prime}, \varphi\left(\mathbf{x}^{\prime}\right)\right)$ and $\tilde{\mathbf{x}}=\left(\mathbf{x}^{\prime}, x_{n}\right)$. The case $x_{n}=\varphi\left(\mathbf{x}^{\prime}\right)$ has already been studied in Proposition 3.1 which ensures that $\mathbf{x} \in \partial \Omega$. Moreover, if $-\varepsilon<x_{n}<-\varepsilon+\sqrt{\varepsilon^{2}-\left\|\mathbf{x}^{\prime}\right\|^{2}}$, then $\tilde{\mathbf{x}} \in B_{\varepsilon}\left(\mathbf{0}^{\prime},-\varepsilon\right) \subseteq \Omega$ and if $-\varepsilon+\sqrt{\varepsilon^{2}-\left\|\mathbf{x}^{\prime}\right\|^{2}} \leqslant x_{n}<\varphi\left(\mathbf{x}^{\prime}\right)$, then apply Lemma 3.2 to get $\tilde{\mathbf{x}} \in \Omega$. To conclude, we proved: $\forall \mathbf{x}^{\prime} \in D_{r}\left(\mathbf{0}^{\prime}\right),-\varepsilon<x_{n}<\varphi\left(\mathbf{x}^{\prime}\right) \Longrightarrow\left(\mathbf{x}^{\prime}, x_{n}\right) \in \Omega$. Similar arguments hold when $\varepsilon>x_{n}>\varphi\left(\mathbf{x}^{\prime}\right)$ and imply $\left(\mathbf{x}^{\prime}, x_{n}\right) \in \mathbb{R}^{n} \backslash \bar{\Omega}$. Finally, note $\mathbf{0}=\mathbf{x}_{\mathbf{0}}=\left(\mathbf{0}^{\prime}, \varphi\left(\mathbf{0}^{\prime}\right)\right)$.

\subsection{The $C^{1,1}$-regularity of the local graph}

Lemma 3.4. The map $f: \alpha \in] 0, \frac{\pi}{2}\left[\mapsto \frac{2 \alpha}{\cos \alpha} \in\right] 0,+\infty[$ is well defined, continuous, surjective and increasing strictly. Hence, it is an homeomorphism and its inverse $f^{-1}$ satisfies for all $\varepsilon>0$ the inequality $f^{-1}(\varepsilon)<\frac{\varepsilon}{2}$.

Proof. The proof is left to the reader.

Proposition 3.5 (Point (i) of Theorem 1.8). Consider $\left.\alpha \in] 0, f^{-1}(\varepsilon)\right]$ where $f$ is defined in Lemma 3.4. Then, we have $C_{\alpha}\left(\mathbf{x},-\mathbf{d}_{\mathbf{x}_{\mathbf{0}}}\right) \subseteq \Omega$ for every point $\mathbf{x} \in B_{\alpha}\left(\mathbf{x}_{\mathbf{0}}\right) \cap \bar{\Omega}$. Hence, $\Omega$ satisfies the $f^{-1}(\varepsilon)$-cone property.

Proof. Set $r=\frac{\sqrt{3}}{2} \varepsilon$ and $\left.\mathcal{C}_{r, \varepsilon}=D_{r}\left(\mathbf{0}^{\prime}\right) \times\right]-\varepsilon, \varepsilon[$. Choose $\left.\alpha \in] 0, f^{-1}(\varepsilon)\right]$ then consider $\mathbf{x}=\left(\mathbf{x}^{\prime}, x_{n}\right) \in B_{\alpha}\left(\mathbf{x}_{\mathbf{0}}\right) \cap \bar{\Omega}$ and $\mathbf{y}=\left(\mathbf{y}^{\prime}, y_{n}\right) \in C_{\alpha}\left(\mathbf{x},-\mathbf{d}_{\mathbf{x}_{0}}\right)$. The proof of the assertion $\mathbf{y} \in \Omega$ is divided in three steps:

- check that $\mathbf{x} \in \mathcal{C}_{r, \varepsilon}$ in order to introduce the point $\tilde{\mathbf{x}}=\left(\mathbf{x}^{\prime}, \varphi\left(\mathbf{x}^{\prime}\right)\right)$ of $\partial \Omega$ that satisfies $x_{n} \leqslant \varphi\left(\mathbf{x}^{\prime}\right)$;

- consider $\tilde{\mathbf{y}}=\left(\mathbf{y}^{\prime}, y_{n}+\varphi\left(\mathbf{x}^{\prime}\right)-x_{n}\right)$ and prove that $\tilde{\mathbf{y}} \in C_{\alpha}\left(\tilde{\mathbf{x}},-\mathbf{d}_{\mathbf{x}_{\mathbf{0}}}\right) \subseteq B_{\varepsilon}\left(\tilde{\mathbf{x}}-\varepsilon \mathbf{d}_{\tilde{\mathbf{x}}}\right) \subseteq \Omega$;

- show that $(\tilde{\mathbf{y}}, \mathbf{y}) \in \mathcal{C}_{r, \varepsilon} \times \mathcal{C}_{r, \varepsilon}$ in order to deduce $y_{n}+\varphi\left(\mathbf{x}^{\prime}\right)-x_{n}<\varphi\left(\mathbf{y}^{\prime}\right)$ and conclude $\mathbf{y} \in \Omega$. 
First, considering the inequality of Lemma 3.4, one obtains: $\max \left(\left\|\mathbf{x}^{\prime}\right\|,\left|x_{n}\right|\right) \leqslant\left\|\mathbf{x}-\mathbf{x}_{\mathbf{0}}\right\|<\alpha \leqslant f^{-1}(\varepsilon)<\frac{\varepsilon}{2}$. Hence, we get $\mathbf{x} \in \bar{\Omega} \cap \mathcal{C}_{r, \varepsilon}$ and from Proposition 3.3, it comes $x_{n} \leqslant \varphi\left(\mathbf{x}^{\prime}\right)$. We set $\tilde{\mathbf{x}}=\left(\mathbf{x}^{\prime}, \varphi\left(\mathbf{x}^{\prime}\right)\right) \in \partial \Omega \cap \mathcal{C}_{r, \varepsilon}$ and observe that $\tilde{\mathbf{x}} \in B_{\alpha \sqrt{2}}\left(\mathbf{x}_{\mathbf{0}}\right)$. Indeed, the inequality of Proposition 3.1 applied to $\tilde{\mathbf{x}}=\left(\mathbf{x}^{\prime}, \varphi\left(\mathbf{x}^{\prime}\right)\right)$ gives:

$$
\left\|\tilde{\mathbf{x}}-\mathbf{x}_{\mathbf{0}}\right\|^{2} \leqslant 2 \varepsilon^{2}-2 \varepsilon \sqrt{\varepsilon^{2}-\left\|\mathbf{x}^{\prime}\right\|^{2}}=\frac{4 \varepsilon^{2}\left\|\mathbf{x}^{\prime}\right\|^{2}}{2 \varepsilon^{2}+2 \varepsilon \sqrt{\varepsilon^{2}-\left\|\mathbf{x}^{\prime}\right\|^{2}}} \leqslant 2\left\|\mathbf{x}^{\prime}\right\|^{2} \leqslant 2\left\|\mathbf{x}-\mathbf{x}_{\mathbf{0}}\right\|^{2}<2 \alpha^{2} .
$$

Then, we prove $C_{\alpha}\left(\tilde{\mathbf{x}},-\mathbf{d}_{\mathbf{x}_{0}}\right) \subseteq B_{\varepsilon}\left(\tilde{\mathbf{x}}-\varepsilon \mathbf{d}_{\tilde{\mathbf{x}}}\right)$ so consider any point $\mathbf{z} \in C_{\alpha}\left(\tilde{\mathbf{x}},-\mathbf{d}_{\mathbf{x}_{0}}\right)$. Using the Cauchy-Schwartz inequality, the one of Proposition 2.5 applied to the points $\tilde{\mathbf{x}} \in \partial \Omega$ and $\mathbf{y}=\mathbf{x}_{\mathbf{0}}$, the fact that $\mathbf{z} \in C_{\alpha}\left(\tilde{\mathbf{x}},-\mathbf{d}_{\mathbf{x}_{0}}\right)$, and the foregoing observation $\tilde{\mathbf{x}} \in B_{\alpha \sqrt{2}}\left(\mathbf{x}_{\mathbf{0}}\right)$, we have successively:

$$
\begin{aligned}
\left\|\mathbf{z}-\tilde{\mathbf{x}}+\varepsilon \mathbf{d}_{\tilde{\mathbf{x}}}\right\|^{2}-\varepsilon^{2} & \leqslant\|\mathbf{z}-\tilde{\mathbf{x}}\|^{2}+2 \varepsilon\|\mathbf{z}-\tilde{\mathbf{x}}\|\left\|\mathbf{d}_{\tilde{\mathbf{x}}}-\mathbf{d}_{\mathbf{x}_{\mathbf{0}}}\right\|+2 \varepsilon\left\langle\mathbf{z}-\tilde{\mathbf{x}} \mid \mathbf{d}_{\mathbf{x}_{\mathbf{0}}}\right\rangle \\
& <\|\mathbf{z}-\tilde{\mathbf{x}}\|^{2}+2\|\mathbf{z}-\tilde{\mathbf{x}}\|\left\|\tilde{\mathbf{x}}-\mathbf{x}_{\mathbf{0}}\right\|-2 \varepsilon\|\mathbf{z}-\tilde{\mathbf{x}}\| \cos \alpha \\
& <\alpha[(1+2 \sqrt{2}) \alpha-2 \varepsilon \cos \alpha]<2 \alpha \cos \alpha(f(\alpha)-\varepsilon) \leqslant 0 .
\end{aligned}
$$

Hence, $\mathbf{z} \in B_{\varepsilon}\left(\tilde{\mathbf{x}}-\varepsilon \mathbf{d}_{\tilde{\mathbf{x}}}\right)$ i.e. $C_{\alpha}\left(\tilde{\mathbf{x}},-\mathbf{d}_{\mathbf{x}_{0}}\right) \subseteq B_{\varepsilon}\left(\tilde{\mathbf{x}}-\varepsilon \mathbf{d}_{\tilde{\mathbf{x}}}\right) \subseteq \Omega$, the last inclusion coming from the $\varepsilon$-ball condition. Moreover, since $\tilde{\mathbf{y}}-\tilde{\mathbf{x}}=\mathbf{y}-\mathbf{x}$ and $\mathbf{y} \in C_{\alpha}\left(\mathbf{x},-\mathbf{d}_{\mathbf{x}_{0}}\right)$, we get $\tilde{\mathbf{y}} \in C_{\alpha}\left(\tilde{\mathbf{x}},-\mathbf{d}_{\mathbf{x}_{0}}\right)$, which ends the proof of $\tilde{\mathbf{y}} \in \Omega$. Finally, we check that $(\mathbf{y}, \tilde{\mathbf{y}}) \in \mathcal{C}_{r, \varepsilon} \times \mathcal{C}_{r, \varepsilon}$. We have successively:

$$
\left\{\begin{array}{l}
\left\|\mathbf{y}^{\prime}\right\| \leqslant\left\|\mathbf{y}^{\prime}-\mathbf{x}^{\prime}\right\|+\left\|\mathbf{x}^{\prime}\right\|<\sqrt{\alpha^{2}-\alpha^{2} \cos ^{2} \alpha}+\alpha=\frac{\alpha}{\cos \alpha}\left(\frac{1}{2} \sin 2 \alpha+\cos \alpha\right) \leqslant \frac{3 f(\alpha)}{4} \leqslant \frac{3 \varepsilon}{4}<r \\
\left|y_{n}\right| \leqslant\left|y_{n}-x_{n}\right|+\left|x_{n}\right| \leqslant\|\mathbf{y}-\mathbf{x}\|+\left\|\mathbf{x}-\mathbf{x}_{\mathbf{0}}\right\|<2 \alpha<f(\alpha) \leqslant \varepsilon \\
\left|y_{n}+\varphi\left(\mathbf{x}^{\prime}\right)-x_{n}\right| \leqslant\|\mathbf{y}-\mathbf{x}\|+\varepsilon-\sqrt{\varepsilon^{2}-\left\|\mathbf{x}^{\prime}\right\|^{2}}<\alpha+\frac{\left\|\mathbf{x}^{\prime}\right\|^{2}}{\varepsilon+\sqrt{\varepsilon^{2}-\left\|\mathbf{x}^{\prime}\right\|^{2}}} \leqslant \alpha\left(1+\frac{\alpha}{\varepsilon}\right)<\frac{3 \alpha}{2} \leqslant \varepsilon .
\end{array}\right.
$$

We used here the triangle inequality, the one of Proposition 3.1, the fact that $\mathbf{y} \in C_{\alpha}\left(\mathbf{x},-\mathbf{d}_{\mathbf{x}_{\mathbf{0}}}\right)$, and $\mathbf{x} \in B_{\alpha}\left(\mathbf{x}_{\mathbf{0}}\right)$. To conclude the proof, use Proposition 3.3 applied to $\tilde{\mathbf{y}} \in \Omega \cap \mathcal{C}_{r, \varepsilon}$ in order to obtain $y_{n}+\varphi\left(\mathbf{x}^{\prime}\right)-x_{n}<\varphi\left(\mathbf{y}^{\prime}\right)$. Since we firstly proved $x_{n} \leqslant \varphi\left(\mathbf{x}^{\prime}\right)$, we have $y_{n}<\varphi\left(\mathbf{y}^{\prime}\right)$. Applying Proposition 3.3 to $\mathbf{y} \in \mathcal{C}_{r, \varepsilon}$, we get $\mathbf{y} \in \Omega$.

Corollary 3.6. The map $\varphi$ of Proposition 3.3 restricted to $D_{\frac{\sqrt{2}}{4} f^{-1}(\varepsilon)}\left(\mathbf{0}^{\prime}\right)$ is $\frac{1}{\tan \left[f^{-1}(\varepsilon)\right]}$-Lipschitz continuous.

Proof. First, we set $\alpha=f^{-1}(\varepsilon), r=\frac{\sqrt{3}}{2} \varepsilon$, and $\tilde{r}=\frac{\sqrt{2}}{4} f^{-1}(\varepsilon)$. Then, we choose any $\left(\mathbf{x}_{+}^{\prime}, \mathbf{x}_{-}^{\prime}\right) \in D_{\tilde{r}}\left(\mathbf{0}^{\prime}\right) \times D_{\tilde{r}}\left(\mathbf{0}^{\prime}\right)$. Since $\tilde{r}<r$ from Lemma 3.4, we can consider $\mathbf{x}_{ \pm}=\left(\mathbf{x}_{ \pm}^{\prime}, \varphi\left(\mathbf{x}_{ \pm}^{\prime}\right)\right)$ and using Proposition 3.1, we get:

$$
\left\|\mathbf{x}_{ \pm}-\mathbf{x}_{\mathbf{0}}\right\|^{2} \leqslant 2 \varepsilon^{2}-2 \varepsilon \sqrt{\varepsilon^{2}-\left\|\mathbf{x}_{ \pm}^{\prime}\right\|^{2}}=\frac{4 \varepsilon^{2}\left\|\mathbf{x}_{ \pm}^{\prime}\right\|^{2}}{2 \varepsilon^{2}+2 \varepsilon \sqrt{\varepsilon^{2}-\left\|\mathbf{x}_{ \pm}^{\prime}\right\|^{2}}} \leqslant 2\left\|\mathbf{x}_{ \pm}^{\prime}\right\|^{2}<2 \tilde{r}^{2}<\alpha^{2} .
$$

Hence, we obtain $\mathbf{x}_{ \pm} \in B_{\alpha}\left(\mathbf{x}_{\mathbf{0}}\right) \cap \partial \Omega$. Moreover, we have: $\left\|\mathbf{x}_{+}-\mathbf{x}_{-}\right\| \leqslant\left\|\mathbf{x}_{+}-\mathbf{x}_{\mathbf{0}}\right\|+\left\|\mathbf{x}_{\mathbf{0}}-\mathbf{x}_{-}\right\|<2 \tilde{r} \sqrt{2}=\alpha$. Finally, applying Proposition 3.5, the points $\mathbf{x}_{ \pm}$cannot belong to the cones $C_{\alpha}\left(\mathbf{x}_{\mp},-\mathbf{d}_{\mathbf{x}_{0}}\right) \subseteq \Omega$ thus we get: $\left|\left\langle\mathbf{x}_{+}-\mathbf{x}_{-} \mid \mathbf{d}_{\mathbf{x}_{0}}\right\rangle\right| \leqslant \cos \alpha\left\|\mathbf{x}_{+}-\mathbf{x}_{-}\right\|=\cos \alpha \sqrt{\left\|\mathbf{x}_{+}^{\prime}-\mathbf{x}_{-}^{\prime}\right\|^{2}+\left|\left\langle\mathbf{x}_{+}-\mathbf{x}_{-} \mid \mathbf{d}_{\mathbf{x}_{\mathbf{0}}}\right\rangle\right|^{2}}$. Consequently, re-arrange these terms to obtain the result of the statement: $\left|\varphi\left(\mathbf{x}_{+}^{\prime}\right)-\varphi\left(\mathbf{x}_{-}^{\prime}\right)\right|=\left|\left\langle\mathbf{x}_{+}-\mathbf{x}_{-} \mid \mathbf{d}_{\mathbf{x}_{\mathbf{0}}}\right\rangle\right| \leqslant \frac{1}{\tan \alpha}\left\|\mathbf{x}_{+}^{\prime}-\mathbf{x}_{-}^{\prime}\right\|$.

Proposition 3.7. Set $\tilde{r}=\frac{\sqrt{2}}{4} f^{-1}(\varepsilon)$. The map $\varphi$ of Proposition 3.3 restricted to $D_{\tilde{r}}\left(\mathbf{0}^{\prime}\right)$ is differentiable and its gradient $\nabla \varphi: D_{\tilde{r}}\left(\mathbf{0}^{\prime}\right) \rightarrow \mathbb{R}^{n-1}$ is L-Lipschitz continuous where $L>0$ depends only on $\varepsilon$. Moreover, $\nabla \varphi\left(\mathbf{0}^{\prime}\right)=\mathbf{0}^{\prime}$ and we have:

$$
\forall \mathbf{a}^{\prime} \in D_{\tilde{r}}\left(\mathbf{0}^{\prime}\right), \quad \nabla \varphi\left(\mathbf{a}^{\prime}\right)=\mathbf{d}_{\mathbf{x}_{\mathbf{0}}}-\frac{1}{\left\langle\mathbf{d}_{\mathbf{a}} \mid \mathbf{d}_{\mathbf{x}_{0}}\right\rangle} \mathbf{d}_{\mathbf{a}}, \quad \text { where } \mathbf{a}=\left(\mathbf{a}^{\prime}, \varphi\left(\mathbf{a}^{\prime}\right)\right) .
$$


Proof. Consider $\left(\mathbf{a}^{\prime}, \mathbf{x}^{\prime}\right) \in D_{\tilde{r}}\left(\mathbf{0}^{\prime}\right) \times D_{\tilde{r}}\left(\mathbf{0}^{\prime}\right)$. From Lemma 3.4, we have $\tilde{r}<r$. Hence, from Proposition 3.3, we can introduce the points $\mathbf{x}=\left(\mathbf{x}^{\prime}, \varphi\left(\mathbf{x}^{\prime}\right)\right)$ and $\mathbf{a}=\left(\mathbf{a}^{\prime}, \varphi\left(\mathbf{a}^{\prime}\right)\right)$. Using the global inequality of Proposition 2.7 applied to $(\mathbf{a}, \mathbf{x}) \in \partial \Omega \times \partial \Omega$ and the Lipschitz continuity of $\varphi$ on $D_{\tilde{r}}\left(\mathbf{0}^{\prime}\right)$ proved in Corollary 3.6, we obtain:

$$
\left|\left(\varphi\left(\mathbf{x}^{\prime}\right)-\varphi\left(\mathbf{a}^{\prime}\right)\right) \mathbf{d}_{\mathbf{a} n}+\left\langle\mathbf{d}_{\mathbf{a}}^{\prime} \mid \mathbf{x}^{\prime}-\mathbf{a}^{\prime}\right\rangle\right|=\left|\left\langle\mathbf{x}-\mathbf{a} \mid \mathbf{d}_{\mathbf{a}}\right\rangle\right| \leqslant \frac{1}{2 \varepsilon}\|\mathbf{x}-\mathbf{a}\|^{2} \leqslant \underbrace{\frac{1}{2 \varepsilon}\left(1+\frac{1}{\tan ^{2}\left[f^{-1}(\varepsilon)\right]}\right)}_{:=\tilde{C}(\varepsilon)>0}\left\|\mathbf{x}^{\prime}-\mathbf{a}^{\prime}\right\|^{2},
$$

where we set $\mathbf{d}_{\mathbf{a} n}=\left\langle\mathbf{d}_{\mathbf{a}} \mid \mathbf{d}_{\mathbf{x}_{\mathbf{0}}}\right\rangle$ and $\mathbf{d}_{\mathbf{a}}^{\prime}=\mathbf{d}_{\mathbf{a}}-\mathbf{d}_{\mathbf{a} n} \mathbf{d}_{\mathbf{x}_{\mathbf{0}}}$. It represents a first-order Taylor expansion of the map $\varphi$ if we can divide the above relation by a strictly positive constant smaller than $\mathbf{d}_{\mathbf{a} n}$. Let us justify this assertion. Apply the inequality of Proposition 2.5 to $\mathbf{x}=\mathbf{a}$ and $\mathbf{y}=\mathbf{x}_{\mathbf{0}}$, then use the inequality of Proposition 3.1 to get:

$$
\mathbf{d}_{\mathbf{a} n}=1-\frac{1}{2}\left\|\mathbf{d}_{\mathbf{a}}-\mathbf{d}_{\mathbf{x}_{\mathbf{0}}}\right\|^{2} \geqslant 1-\frac{1}{2 \varepsilon^{2}}\left\|\mathbf{a}-\mathbf{x}_{\mathbf{0}}\right\|^{2} \geqslant 1-\frac{\varepsilon-\sqrt{\varepsilon^{2}-\left\|\mathbf{a}^{\prime}\right\|^{2}}}{\varepsilon}=1-\frac{\left\|\mathbf{a}^{\prime}\right\|^{2}}{\varepsilon\left(\varepsilon+\sqrt{\varepsilon^{2}-\left\|\mathbf{a}^{\prime}\right\|^{2}}\right)}>\underbrace{1-\frac{\tilde{r}^{2}}{\varepsilon^{2}}}_{:=C(\varepsilon)>0} .
$$

Hence, we obtain the announced first-order Taylor expansion: $\left|\varphi\left(\mathbf{x}^{\prime}\right)-\varphi\left(\mathbf{a}^{\prime}\right)+\left\langle\frac{\mathbf{d}_{\mathbf{a}}^{\prime}}{\mathbf{d}_{\mathbf{a} n}} \mid \mathbf{x}^{\prime}-\mathbf{a}^{\prime}\right\rangle\right| \leqslant \frac{\tilde{C}(\varepsilon)}{C(\varepsilon)}\left\|\mathbf{x}^{\prime}-\mathbf{a}^{\prime}\right\|^{2}$. Therefore, the map $\varphi$ is differentiable at any point $\mathbf{a}^{\prime} \in D_{\tilde{r}}\left(\mathbf{0}^{\prime}\right)$ and its gradient is the one given in the statement. Moreover, for every $\left(\mathbf{a}^{\prime}, \mathbf{x}^{\prime}\right) \in D_{\tilde{r}}\left(\mathbf{0}^{\prime}\right) \times D_{\tilde{r}}\left(\mathbf{0}^{\prime}\right)$, we have successively:

$$
\begin{aligned}
\left\|\nabla \varphi\left(\mathbf{x}^{\prime}\right)-\nabla \varphi\left(\mathbf{a}^{\prime}\right)\right\| & \leqslant\left|\frac{1}{\mathbf{d}_{\mathbf{a} n}}-\frac{1}{\mathbf{d}_{\mathbf{x} n}}\right|\left\|\mathbf{d}_{\mathbf{x}}^{\prime}\right\|+\left|\frac{1}{\mathbf{d}_{\mathbf{a} n}}\right|\left\|\mathbf{d}_{\mathbf{a}}^{\prime}-\mathbf{d}_{\mathbf{x}}^{\prime}\right\| \leqslant \frac{\left|\mathbf{d}_{\mathbf{x} n}-\mathbf{d}_{\mathbf{a} n}\right|}{\tilde{C}(\varepsilon)^{2}}+\frac{\left\|\mathbf{d}_{\mathbf{a}}-\mathbf{d}_{\mathbf{x}}\right\|}{\tilde{C}(\varepsilon)} \\
& \leqslant \frac{1}{\varepsilon \tilde{C}(\varepsilon)}\left(1+\frac{1}{\tilde{C}(\varepsilon)}\right)\|\mathbf{x}-\mathbf{a}\| \leqslant \frac{1}{\varepsilon \tilde{C}(\varepsilon)}\left(1+\frac{1}{\tilde{C}(\varepsilon)}\right) \sqrt{1+\frac{1}{\tan ^{2}\left[f^{-1}(\varepsilon)\right]}\left\|\mathbf{x}^{\prime}-\mathbf{a}^{\prime}\right\|,}
\end{aligned}
$$

where we used the inequality of Proposition 2.5 applied to $\mathbf{x}$ and $\mathbf{y}=\mathbf{a}$, and the Lipschitz continuity of $\varphi$. Hence, the map $\nabla \varphi: \mathbf{a}^{\prime} \in D_{\tilde{r}}\left(\mathbf{0}^{\prime}\right) \mapsto \nabla \varphi\left(\mathbf{a}^{\prime}\right)$ is Lipschitz continuous and its modulus only depends on $\varepsilon$.

Corollary 3.8 (Point (ii) and (iii) of Theorem 1.8). The unit vector $\mathbf{d}_{\mathbf{x}_{\mathbf{0}}}$ is the outer normal to $\partial \Omega$ at $\mathbf{x}_{\mathbf{0}}$. In particular, the $\frac{1}{\varepsilon}$-Lipschitz map $\mathbf{d}: \mathbf{x} \mapsto \mathbf{d}_{\mathbf{x}}$ of Proposition 2.5 is the Gauss map of the $C^{1,1}$-hypersurface $\partial \Omega$.

Proof. Consider $\left.\varphi: D_{\tilde{r}}\left(\mathbf{0}^{\prime}\right) \rightarrow\right]-\varepsilon, \varepsilon\left[\right.$ whose $C^{1,1}$-regularity comes from Proposition 3.7. Define the $C^{1,1}$-map $X: D_{\tilde{r}}\left(\mathbf{0}^{\prime}\right) \rightarrow \partial \Omega$ by $X\left(\mathbf{x}^{\prime}\right)=\left(\mathbf{x}^{\prime}, \varphi\left(\mathbf{x}^{\prime}\right)\right)$. Consider any $\mathbf{x}^{\prime} \in D_{\tilde{r}}\left(\mathbf{0}^{\prime}\right)$. Let $\left(e_{k}\right)_{1 \leqslant k \leqslant n-1}$ refer to the first vectors of our local basis. The tangent plane of $\partial \Omega$ at $X\left(\mathbf{x}^{\prime}\right)$ is spanned by the vectors $\partial_{k} X\left(\mathbf{x}^{\prime}\right)=e_{k}+\left(\mathbf{0}^{\prime}, \partial_{k} \varphi\left(\mathbf{x}^{\prime}\right)\right)$. Consider $\mathbf{u}=\left(u_{1}, \ldots, u_{n}\right)$ a normal vector to this hyperplane. Thus, it is orthogonal to this $(n-1)$ vectors: $\left\langle\mathbf{u} \mid \partial_{k} X\left(\mathbf{x}^{\prime}\right)\right\rangle=0 \Leftrightarrow u_{k}+u_{n} \partial_{k} \varphi\left(\mathbf{x}^{\prime}\right)=0 \Leftrightarrow u_{k}=\frac{u_{n}}{\mathbf{d}_{\mathbf{x}_{n}}} \mathbf{d}_{\mathbf{x} k}$. Hence, we get $\mathbf{u}=\frac{u_{n}}{\mathbf{d}_{\mathbf{x}_{n}}} \mathbf{d}_{\mathbf{x}}$ so $\mathbf{u}$ is collinear to $\mathbf{d}_{\mathbf{x}}$. Now, if we impose that $\mathbf{u}$ points outwards $\Omega$ and $\|\mathbf{u}\|=1$, then we get $\mathbf{u}=\mathbf{d}_{\mathbf{x}}$.

\subsection{The compact case: when $C^{1,1}$-regularity implies the uniform ball condition}

Proposition 3.9 (Jordan-Brouwer separation theorem). If $\mathcal{S}$ is a compact connected topological hypersurface of $\mathbb{R}^{n}$, then $\mathbb{R}^{n} \backslash \mathcal{S}$ has exactly two connected components whose boundary is $\mathcal{S}$ and only one is bounded. Moreover, $\mathcal{S}$ is orientable in the algebraic sense which coincides with the usual sense once $\mathcal{S}$ has $C^{1}$-regularity.

Proof. We refer to [14] for a proof (VIII 8.15 page 301) and an algebraic definition of orientability (VIII 1-2).

Corollary 3.10. Consider a compact topological hypersurface $\mathcal{S}$ of $\mathbb{R}^{n}$. Then, there exists a unique bounded open subset $\Omega$ of $\mathbb{R}^{n}$ such that $\partial \Omega=\mathcal{S}$. Moreover, the hypersurface $\mathcal{S}$ is orientable in the algebraic sense.

Proof. We only sketch the proof. Note $\mathcal{S}$ has a finite number of connected components, otherwise we get from compactness an accumulation point that cannot be homeomorphic to the locally connected space $\mathbb{R}^{n-1}$. Apply Proposition 3.9 on each connected component. As they cannot intersect, one can build $\Omega$ such that $\partial \Omega=\mathcal{S}$. 
Proposition 3.11 (Proof of Theorem 1.9). Consider any non-empty compact $C^{1,1}$-hypersurface $\mathcal{S}$ of $\mathbb{R}^{n}$. Then, $\mathcal{S}$ is orientable in the usual differentiable sense and there exists a unique bounded open subset $\Omega$ of $\mathbb{R}^{n}$ such that $\partial \Omega=\mathcal{S}$. Moreover, there exists a real number $\varepsilon>0$ such that $\Omega$ satisfies the $\varepsilon$-ball condition.

Proof. Consider a non-empty compact $C^{1,1}$-hypersurface $\mathcal{S}$ of $\mathbb{R}^{n}$. Apply Corollary 3.10 to get its orientability and the existence of $\Omega$. Note the $C^{1,1}$-regularity has not been used so far. Consider any $\mathbf{x}_{\mathbf{0}} \in \partial \Omega$ and its local frame as in Definition 1.5. First, we have for any $\left(\mathbf{x}^{\prime}, \mathbf{y}^{\prime}\right) \in D_{r}\left(\mathbf{0}^{\prime}\right) \times D_{r}\left(\mathbf{0}^{\prime}\right)$ with $g: t \in[0,1] \mapsto \varphi\left(\mathbf{x}^{\prime}+t\left(\mathbf{y}^{\prime}-\mathbf{x}^{\prime}\right)\right)$ :

$$
\begin{aligned}
\left|\varphi\left(\mathbf{y}^{\prime}\right)-\varphi\left(\mathbf{x}^{\prime}\right)-\left\langle\nabla \varphi\left(\mathbf{x}^{\prime}\right) \mid \mathbf{y}^{\prime}-\mathbf{x}^{\prime}\right\rangle\right| & =\left|g(1)-g(0)-g^{\prime}(0)\right|=\left|\int_{0}^{1}\left[g^{\prime}(t)-g^{\prime}(0)\right] d t\right| \leqslant \int_{0}^{1}\left|g^{\prime}(t)-g^{\prime}(0)\right| d t \\
& \leqslant \int_{0}^{1}\left\|\nabla \varphi\left(\mathbf{x}^{\prime}+t\left(\mathbf{y}^{\prime}-\mathbf{x}^{\prime}\right)\right)-\nabla \varphi\left(\mathbf{x}^{\prime}\right)\right\|\left\|\mathbf{y}^{\prime}-\mathbf{x}^{\prime}\right\| d t \leqslant \frac{L}{2}\left\|\mathbf{y}^{\prime}-\mathbf{x}^{\prime}\right\|^{2} .
\end{aligned}
$$

Then, we set $\varepsilon_{0}=\min \left(\frac{1}{L}, \frac{r}{3}, \frac{a}{3}\right)$ and consider any $\mathbf{x} \in B_{\varepsilon_{0}}\left(\mathbf{x}_{\mathbf{0}}\right) \cap \partial \Omega$. Since $\varepsilon_{0} \leqslant \min (r, a)$, we get $\mathbf{x}=\left(\mathbf{x}^{\prime}, \varphi\left(\mathbf{x}^{\prime}\right)\right)$ for a certain $\mathbf{x}^{\prime} \in D_{r}\left(\mathbf{0}^{\prime}\right)$. We introduce the notation $\left.\left.\mathbf{d}_{\mathbf{x} n}=\left(1+\left\|\nabla \varphi\left(\mathbf{x}^{\prime}\right)\right\|^{2}\right)^{-\frac{1}{2}} \in\right] 0,1\right]$ and $\mathbf{d}_{\mathbf{x}}^{\prime}=-\mathbf{d}_{\mathbf{x} n} \nabla \varphi\left(\mathbf{x}^{\prime}\right)$ so that $\mathbf{d}_{\mathbf{x}}:=\left(\mathbf{d}_{\mathbf{x}}^{\prime}, \mathbf{d}_{\mathbf{x} n}\right)$ is a unit vector. Now, let us show that $\Omega$ satisfy the $\varepsilon_{0}$-ball condition at the point $\mathbf{x}$ so choose any $\mathbf{y} \in B_{\varepsilon_{0}}\left(\mathbf{x}+\varepsilon_{0} \mathbf{d}_{\mathbf{x}}\right) \subseteq B_{2 \varepsilon_{0}}(\mathbf{x}) \subseteq B_{3 \varepsilon_{0}}\left(\mathbf{x}_{\mathbf{0}}\right)$. Since $3 \varepsilon_{0} \leqslant \min (r, a)$, there exists $\mathbf{y}^{\prime} \in D_{r}\left(\mathbf{0}^{\prime}\right)$ and $\left.y_{n} \in\right]-a, a\left[\right.$ such that $\mathbf{y}=\left(\mathbf{y}^{\prime}, y_{n}\right)$. Moreover, we have the characterization: $\mathbf{y} \in \mathbb{R}^{n} \backslash \bar{\Omega}$ if and only if $y_{n}>\varphi\left(\mathbf{y}^{\prime}\right)$. Observing that $\left\|\mathbf{y}-\mathbf{x}-\varepsilon_{0} \mathbf{d}_{\mathbf{x}}\right\|<\varepsilon_{0} \Leftrightarrow \frac{1}{2 \varepsilon_{0}}\|\mathbf{y}-\mathbf{x}\|^{2}<\left\langle\mathbf{y}-\mathbf{x} \mid \mathbf{d}_{\mathbf{x}}\right\rangle$, we obtain successively:

$$
\begin{aligned}
y_{n}-\varphi\left(\mathbf{y}^{\prime}\right) & =\frac{1}{\mathbf{d}_{\mathbf{x} n}}\left[\mathbf{d}_{\mathbf{x} n}\left(y_{n}-\varphi\left(\mathbf{x}^{\prime}\right)\right)+\left\langle\mathbf{d}_{\mathbf{x}}^{\prime} \mid \mathbf{y}^{\prime}-\mathbf{x}^{\prime}\right\rangle-\left\langle\mathbf{d}_{\mathbf{x}}^{\prime} \mid \mathbf{y}^{\prime}-\mathbf{x}^{\prime}\right\rangle+\mathbf{d}_{\mathbf{x} n}\left(\varphi\left(\mathbf{x}^{\prime}\right)-\varphi\left(\mathbf{y}^{\prime}\right)\right)\right] \\
& =\frac{1}{\mathbf{d}_{\mathbf{x} n}}\left\langle\mathbf{y}-\mathbf{x} \mid \mathbf{d}_{\mathbf{x}}\right\rangle-\varphi\left(\mathbf{y}^{\prime}\right)+\varphi\left(\mathbf{x}^{\prime}\right)+\left\langle\nabla \varphi\left(\mathbf{x}^{\prime}\right) \mid \mathbf{y}^{\prime}-\mathbf{x}^{\prime}\right\rangle>\frac{\|\mathbf{y}-\mathbf{x}\|^{2}}{2 \varepsilon_{0} \mathbf{d}_{\mathbf{x} n}}-\frac{L}{2}\left\|\mathbf{y}^{\prime}-\mathbf{x}^{\prime}\right\|^{2}
\end{aligned}
$$

Consequently, we get $y_{n}-\varphi\left(\mathbf{y}^{\prime}\right)>\frac{1}{2 \mathbf{d}_{\mathbf{x}}}\left\|\mathbf{y}^{\prime}-\mathbf{x}^{\prime}\right\|^{2}\left(\frac{1}{\varepsilon_{0}}-L\right) \geqslant 0$ thus $\mathbf{y} \notin \bar{\Omega}$ and we proved $B_{\varepsilon_{0}}\left(\mathbf{x}+\varepsilon_{0} \mathbf{d}_{\mathbf{x}}\right) \subseteq \mathbb{R}^{n} \backslash \bar{\Omega}$. Similarly, we can obtain $B_{\varepsilon_{0}}\left(\mathbf{x}-\varepsilon_{0} \mathbf{d}_{\mathbf{x}}\right) \subseteq \Omega$. Hence, for any $\mathbf{x}_{\mathbf{0}} \in \partial \Omega$, there exists $\varepsilon_{0}>0$ such that $\Omega \cap B_{\varepsilon_{0}}\left(\mathbf{x}_{\mathbf{0}}\right)$ satisfies the $\varepsilon_{0}$-ball condition. Finally, as $\partial \Omega$ is compact, it is included in a finite reunion of such balls $B_{\varepsilon_{0}}\left(\mathbf{x}_{\mathbf{0}}\right)$. Define $\varepsilon>0$ as the minimum of this finite number of $\varepsilon_{0}$ and $\Omega$ will satisfy the $\varepsilon$-ball property as required.

\section{REFERENCES}

[1] P. B. Canham, The minimum energy of bending as a possible explanation of the biconcave shape of the human red blood cell, Journal of Theoretical Biology, 26 (1), 61-81, January 1970.

[2] W. Helfrich, Elastic properties of lipid bilayers: theory and possible experiments, Zeit. für. Naturfor., 28 (C), 693-703, 1973.

[3] U. Seifert, K. Berndl, and R. Lipowsky, Shape transformations of vesicles: phase diagram for spontaneous-curvature and bilayer-coupling models, Physical Review A, 44 (2), 1182-1202, July 1991.

[4] G. Bellettini, G. Dal Maso, and M. Paolini, Semicontinuity and relaxation properties of curvature depending functional in 2D, Annali della Scuola Normale Superiore di Pisa, Classe di Scienze 4ième série, 20 (2), 247-297, 1993.

[5] R. Choksi and M. Veneroni, Global minimizers for the doubly-constrained Helfrich energy: the axisymmetric case, Calculus of Variations and Partial Differential Equations, August 2012.

[6] T. J. Willmore, Total Curvature in Riemannian Geometry, Mathematics and its Applications, Ellis Horwood, 1982.

[7] L. Simon, Existence of surfaces minimizing the Willmore functional, Comm. in Anal. and Geom., 1 (2), 281-326, 1993.

[8] M. Bauer and E. Kuwert, Existence of Minimizing Willmore Surfaces of Prescribed Genus, International Mathematics Research Notices, 1 (10), 553-576, 2003.

[9] J. Schygulla, Willmore Minimizers with Prescribed Isoperimetric Ratio, Arch. Rat. Mech. and Anal., 203 (3), 901-941, 2012.

[10] D. Chenais, Homéomorphisme entre ouverts lipschitziens, Annali di Matematica Pura ed Applicata, 118, 343-398, 1978.

[11] J. Dalphin, Uniform ball property and existence of optimal shapes for a wide class of functionals, to appear.

[12] H. Federer, Curvature measures, Transaction of the American Mathematical Society, 93, 418-491, 1959.

[13] B. Z. Guo AND D. H. YANG, Some compact classes of open sets under Hausdorff distance and application to shape optimization, SIAM Journal of Control Optimization, 50 (1), 222-242, January 2012.

[14] A. Dold, Lectures on algebraic topology, Grundlehren der Mathematischen Wissenschaften, 200, Springer, 1972. 